\title{
3D Bilderfassung und Analyse in der Plastischen Chirurgie mit Smartphone und Tablet: eine Alternative zu professionellen Systemen?
}

\author{
3D-Imaging and Analysis for Plastic Surgery by Smartphone and Tablet: An \\ Alternative to Professional Systems?
}

\author{
Autoren \\ K. C. Koban*, S. Leitsch*, T. Holzbach, E. Volkmer, P. M. Metz, R. E. Giunta \\ Institut \\ Handchirurgie, Plastische Chirurgie, Ästhetische Chirurgie, Klinikum der Ludwig-Maximilians Universität München, \\ München
}

\section{Schlüsselwörter \\ - 3D \\ - Photogrammetrie \\ Oberflächenerfassung \\ - Smartphone \\ - Applikation}

\section{Key words \\ - 3D-imaging \\ - photogrammetry \\ - surface scanning \\ - smartphones \\ - applications}

eingereicht 17.2.2014 akzeptiert $\quad 3.3 .2014$

\section{Bibliografie DOI http://dx.doi.org/ 10.1055/s-0034-1371822 Handchir Mikrochir Plast Chir 2014; 46: 97-104 (c) Georg Thieme Verlag KG Stuttgart · New York ISSN 0722-1819}

Korrespondenzadresse Dr. med. Sebastian Leitsch Handchirurgie, Plastische Chirurgie

Äshetische Chirurgie Klinikum der Ludwig-Maximilians Universität München Pettenkofer 8a 80336 München Sebastian.Leitsch@med.unimuenchen.de

\section{Zusammenfassung}

\section{$\nabla$}

Hintergrund: Eine neue Alternative zu professionellen dreidimensionalen (3D) Kamerasystemen nutzt Digitalfotos von Smartphones zur 3D Oberflächenerfassung. In dieser Studie untersuchten wir deren Anwendung mit unterschiedlichen Einstellungen. Es wurden die Genauigkeit der 3D Rekonstruktion gegenüber der manuellen Maßbandmessung und einem etablierten 3D Kamerasystem verglichen, sowie die benötigte Zeit beider 3D Systeme.

Material und Methoden: Es wurde ein Puppenkopfmodel mit 21 Landmarken im Gesichtsbereich für standardisierte 3D Aufnahmen genutzt. Das mit dem Vectra 3D Imaging System ${ }^{\circledR}$ erstellte 3D Model diente als Referenz gegenüber den Catch ${ }^{\circledR}$ Modellen. Die Applikation Autodesk 123d Catch ${ }^{\circledR}$ nutzte 16, 12, 9, 6 und 3 Digitalfotos eines Apple ${ }^{\circledR}$ iPhone $4 s^{\circledR}$ und iPad ${ }^{\circledR}$ der 3ten Generation zur 3D Erfassung des Puppenkopfes. Im ersten Schritt der Genauigkeitsanalyse wurden insgesamt 42 Distanzen durch manuelle Maßbandmessung und beide digitalen Systeme bestimmt und verglichen. Im Weiteren wurde die Genauigkeit der 3D Oberfläche der durch Catch $^{\circledR}$ generierten 3D Modelle gegenüber dem Vectra ${ }^{\circledR}$ Referenzmodel anhand von ästhetischen Einheiten untersucht. Zusätzlich wurden beide 3D Systeme hinsichtlich ihrer Aufnahme- und Verarbeitungszeit evaluiert.

Ergebnisse: Es zeigten sich keine signifikanten Unterschiede in den Distanzen $(p>0,05)$ zwischen der manuellen Messung und den digitalen Messungen des Catch ${ }^{\circledR}$ und Vectra ${ }^{\circledR}$ Systems. Die 3D Rekonstruktion durch Catch $^{\circledR}$ zeigte bei der Nutzung von 16, 12 und 9 Bildern im Oberflächenvergleich zum Vectra ${ }^{\circledR}$ Referenzmodel eine zufriedenstellende Genauigkeit. 6 und 3 Bilder führten zu einer hohen Abweichung. Laterale Ge-

* Beide aufgeführten Personen teilen sich die Autorenschaft gleichmäßig.

\section{Abstract}

$\nabla$

Background: A new approach of using photographs from smartphones for three-dimensional (3D) imaging was introduced besides the standard high quality 3D camera systems. In this work, we investigated different capture preferences and compared the accuracy of this 3D reconstruction method with manual tape measurement and an established commercial 3D camera system.

Methods: The facial region of one plastic mannequin head was labelled with 21 landmarks. A 3D reference model was captured with the Vectra 3D Imaging System ${ }^{\circledR}$. In addition, 3D imaging was executed with the Autodesk 123d Catch ${ }^{\circledR}$ application using 16, 12, 9, 6 and 3 pictures from Apple ${ }^{\circledR}$ iPhone $4 s^{\circledR}$ and iPad ${ }^{\circledR} 3^{\text {rd }}$ generation. The accuracy of 3D reconstruction was measured in 2 steps. First, 42 distance measurements from manual tape measurement and the 2 digital systems were compared. Second, the surface-to-surface deviation of different aesthetic units from the Vectra ${ }^{\circledR}$ reference model to Catch ${ }^{\circledR}$ generated models was analysed. For each 3D system the capturing and processing time was measured.

Results: The measurement showed no significant $(p>0.05)$ difference between manual tape measurement and both digital distances from the Catch $^{\circledR}$ application and Vectra ${ }^{\circledR}$. Surface-to-surface deviation to the Vectra ${ }^{\circledR}$ reference model showed sufficient results for the 3D reconstruction of Catch $^{\circledR}$ with 16,12 and 9 picture sets. Use of 6 and 3 pictures resulted in large deviations. Lateral aesthetic units showed higher deviations than central units. Catch ${ }^{\circledR}$ needed 5 times longer to capture and compute 3D models (average 10 min vs. 2 min).

Conclusion: The Autodesk 123d Catch ${ }^{\circledR}$ computed models suggests good accuracy of the 3D reconstruction for a standard mannequin model, in comparison to manual tape measurement and 
sichtsbereiche zeigten insgesamt eine höhere Abweichung als zentrale Bereiche. Catch ${ }^{\circledR}$ benötigt im Vergleich die 5-fache Zeit (durchschnittlich $10 \mathrm{~min}$ vs. $2 \mathrm{~min}$ ) bis zu einem fertigen 3D Model.

Schlussfolgerung: Insgesamt konnten durch den standardisierten Ansatz mit der mobilen Applikation 123 Catch $^{\circledR}$ am Puppenmodel eine zufriedenstellende Genauigkeit der 3D Rekonstruktion gegenüber der manuellen Distanzmessung und dem Oberflächenvergleich mit einem Vectra ${ }^{\circledR}$ Referenzmodel gezeigt werden. Hauptproblem ist derzeit die längere Akquisitionszeit. Weitere Studien werden benötigt, um die Anwendbarkeit und Genauigkeit bei der 3D Erfassung von Probanden zu untersuchen. In Zukunft könnten mobile Anwendungen eine kostengünstigere Alternative gegenüber den derzeit statischen und teuren 3D Kamerasystemen in der Plastischen Chirurgie darstellen.

\section{Einleitung}

Kamerasysteme zur 3-dimensionalen (3D) strahlungsfreien Oberflächenerfassung werden zunehmend auch außerhalb großer Universitätskliniken zur Erweiterung des Arzt-PatientenGespräches in der Plastischen Chirurgie eingesetzt. Obwohl das Verfahren zur 3D Rekonstruktion aus 2D Bildern (digitale Photogrammetrie) seit Jahrzehnten bekannt ist [1-3], zeigten sich entscheidende Entwicklungen in den letzten Jahren sowohl in der immer höheren Präzision der Geräte, als auch dem erweiterten Einsatzspektrum[4,5]. 3D Kamerasysteme bieten heutzutage eine umfassende Dokumentation und Planung unterschiedlicher formverändernder Eingriffe und werden insbesondere im Bereich der Mund-Kiefer-Gesichts-Chirurgie und der Plastischen, Rekonstruktiven und Ästhetischen Chirurgie genutzt. Initial mit einem hohen technischen Aufwand verbunden und in wenigen ausgewählten Forschergruppen angewandt, sind derzeit verschiede Kamerasysteme auf dem Markt zu erwerben. Die Bedienung und Technik wurde immer weiter der klinischen Umsetzbarkeit angepasst, wodurch heutige automatische 3D Systeme leicht zu bedienen sind und auch durch niedergelassene Chirurgen in der Patientenkonsultation eingesetzt werden.

Obwohl zunehmend kleinere, mobile Systeme entwickelt werden, sind die bereits etablierten Geräte, wie das in unserer Klinik eingesetzte Vectra 3D Surface Imaging System ${ }^{\circledR}$ (Canfield Sci, NJ, USA) [6], noch relativ groß, unbeweglich und zumeist auf wenige Körperteile wie Gesicht, Brust und Bauch spezialisiert. Dabei stellen hohe Anschaffungskosten immer noch die größte Hemmschwelle zum Erwerb der nützlichen Geräte dar. Verschiedene Ansätze mit kostengünstigeren Alternativen wurden bereits verfolgt, darunter auch die alternative Nutzung einer TiefensensorKamera eines bekannten Multimediasystems [7,8].

Einen anderen Ansatz zur Ergänzung des Arzt-Patienten-Gespräches verfolgen kostenpflichtige Softwarelösungen mit einer $3 D$ Verarbeitung über das Internet $[9,10]$. Hierbei werden durch den Arzt aufgenommene Digitalfotos von Gesicht oder Brust seiner Patienten zur online 3D Rekonstruktion an Firmen übermittelt. Durch den Nutzen von Digitalkameras entfällt somit die Anschaffung eines 3D Systems.

Eine neue kostenlose Variante bietet die Firma Autodesk ${ }^{\circledR}$ (Autodesk Inc., San Rafael, CA, USA) mit ihrer Applikation 123d Catch ${ }^{\circledR}$ $\left(\right.$ kurz Catch $^{\circledR}$ ) für die mobile Geräte Apple ${ }^{\circledR}$ iPhone $^{\circledR}$ und iPad $^{\circledR}$ (Apple Inc., Cupertino, CA, USA). Diese Anwendung nutzt die mit dem Smartphone oder Tablet geschossenen Digitalfotos eines Objekts zur Berechnung eines 3D Models. Die schwache Rechen- the surface-to-surface analysis with a 3D reference model. However, the prolonged capture time with multiple pictures is prone to errors. Further studies are needed to investigate its application and quality in capturing volunteer models. Soon mobile applications may offer an alternative for plastic surgeons to today's cost intensive, stationary 3D camera systems.

leistung der mobilen Geräte wird umgangen, indem die geschossenen Bilder automatisch über das Internet auf externe Rechenzentren hochgeladen und dort verarbeitet werden. Der Anwender erhält nach einer gewissen Verarbeitungszeit ein fertiges 3D Model seiner Aufnahmen zurück. Entwickelt für das schnelle und kostengünstige 3D Erfassen von Objekten für den aufstrebenden Bereich des 3D Drucks, lassen sich mit alltäglichen Smartphones und Tablets auch Personen erfassen. Im Sinne der zunehmenden Integrierung von mobilen Endgeräten [11,12], wie Smartphones und Tablets, im klinischen Alltag, würde sich eine solche Technik schneller etablieren können, sofern der Datenschutz gewährleistet werden kann.

Unsere Arbeitsgruppe hat sich als Ziel dieser Arbeit mit der Evaluierung der mobilen Applikation Catch ${ }^{\circledR}$ zur Erfassung von Objekten unter standardisierten Bedingungen befasst. Es wurden die Genauigkeit der 3D Rekonstruktion anhand eines Vergleichs mit der manuellen Maßbandmessung, sowie dem etablierten Vectra System ${ }^{\circledR}$ untersucht. Zusätzlich wurde die benötigte Zeit zwischen den zwei 3D Rekonstruktionsverfahren verglichen.

\section{Material und Methoden \\ $\nabla$}

\section{Datenerhebung}

Als Objekt für standardisierte Aufnahmen diente ein unbewegliches Puppenkopfmodel auf einem ebenen Tisch. Dieses wurde dezent gepudert, um Lichtstreuungsartefakt während der Aufnahmen zu reduzieren. Sämtliche 3D Aufnahmen wurden in einem gleichmäßig hellen Raum und vor einem blauen Hintergrund aufgenommen.

2 verschiedene Systeme zur 3D Erfassungen wurden für diese Studie genutzt.

Eine hochauflösende 3D Aufnahme des Puppenkopfes wurde mit dem kalibrierten Vectra Volumetric 3D Surface Imaging System ${ }^{\circledR}$ (Canfield Sci, NJ, USA) erfasst. Dieses 3D Model diente später als digitale Referenz gegenüber den mit Catch ${ }^{\circledR}$ generierten Modellen.

Im Weiteren wurden mit einem Apple ${ }^{\circledR} \operatorname{iPad}^{\circledR}$ der 3ten Generation und einem Apple ${ }^{\circledR}$ iPhone $4 \mathrm{~s}^{\circledR}$ insgesamt 16 Bilder aus $50 \mathrm{~cm}$ Entfernung rund um das Puppenkopfmodel aufgenommen ( $\bullet$ Abb. 1). Die auf den beiden Geräten installierte mobile Anwendung 123d Catch $^{\circledR}$ (Softwareversion 1.0.3) nutzt diese Bilder zur automatischen Berechnung von 3D Modellen und benötigt keine vorherige Kalibrierung. 
Zur Untersuchung der Auswirkung von 3 verschiedenen Qualitätsstufen der Berechnung, sowie einer geringeren Anzahl an Fotos auf die 3D Rekonstruktion nutzten wir die Desktop Variante von Catch $^{\circledR}$ (Version v.2.2.3.377). Mit dieser wurden schrittweise die Anzahl der Fotos zur 3D Verarbeitung auf je 12, 9, 6 und 3 Bilder reduziert. Alle Catch ${ }^{\circledR}$ Modelle wurden zusätzlich in hoher Qualität (HQ), Standard Qualität (SQ) und niedriger Qualität (LQ) generiert.

\section{Genauigkeitsanalyse}

Die Genauigkeit der 3D Rekonstruktion durch Catch ${ }^{\circledR}$ wurde in 2 Schritten bestimmt.

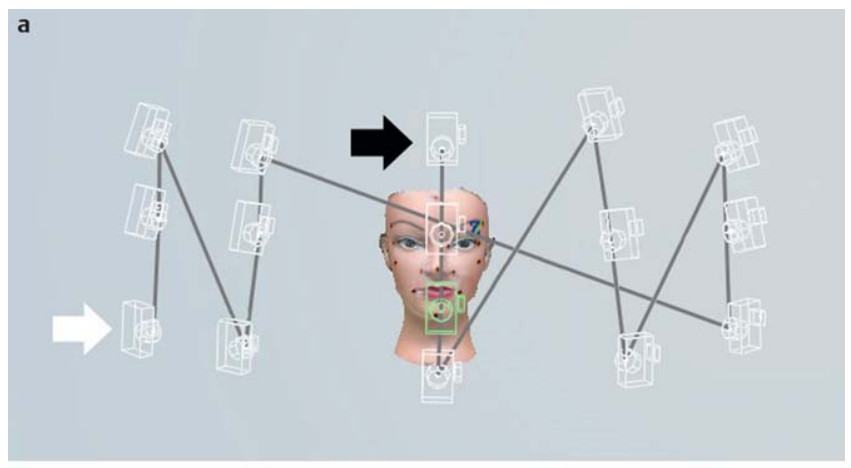

b

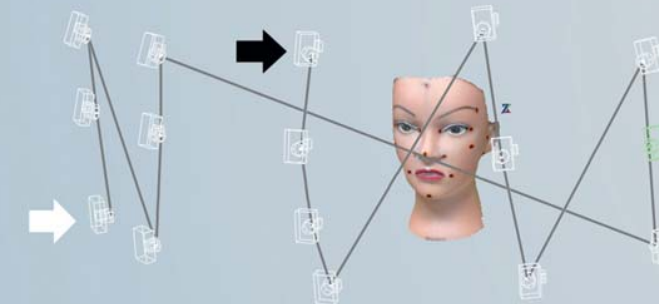

Abb. 1 a + b: Aufnahmepositionen. In einem $50 \mathrm{~cm}$ Abstand wurden dem schwarzen zum weißen Pfeil folgend insgesamt 16 Bilder des Puppenkopfes aufgenommen, davon 4 frontal, sowie pro Seite je 3 Bilder von $30^{\circ}$ medio-lateral und 3 Bilder von $60^{\circ}$ lateral. In jedem Bild wurde das Gesicht fokussiert, wobei der Kopf $50 \%$ des Bildes eingenommen hat. Abbildungen der Anwendung Catch ${ }^{\circledR}$ entnommen.
Zunächst wurde die Abweichung von Distanzmessung auf den 3D Modeloberflächen gegenüber der manuellen Maßbandmessung auf der Puppenkopfoberfläche bestimmt.

Dazu wurden initial 21 Landmarken ( $\bullet$ Abb. 2) im Gesichtsbereich des Puppenmodels gesetzt [13-15] (Stirn, Augenbrauen lateral, äußere Augenwinkel, innere Augenwinkel, Nasenspitze, Nasenflügel, Mundwinkel, Kinn, Jochbeine, Kieferwinkel, Tragus, Ohrläppchen). Aus diesen wurden 42 Distanzen definiert ( $\odot$ Tab. 1), die manuell per Maßband und digital mittels MIRROR $^{\circledR}$ (Canfield Sci., NJ, USA) Software auf den 3D Modellen vermessen wurden. Zur digitalen Distanzmessung nutzten wir das Vectra ${ }^{\circledR}$ Referenzmodell, sowie 3 Catch ${ }^{\circledR}$ Modelle, welche mit der maximalen Anzahl an 16 Bildern in den 3 Qualitätsstufen HQ SQ, LQ generiert wurden.

3 Untersucher wiederholten jeweils 3-mal die manuelle Maßbandmessung und die digitalen Distanzmessungen an der Modeloberfläche. Wir definierten den endgültigen Wert als den Mittelwert der 3 Messungen für jede Distanz. Die Genauigkeit der Distanzmessungen wurde definiert als die Abweichung der digitalen Messungen beider 3D Systeme von der manuellen Maßbandmessung.

Im zweiten Schritt wurde die Genauigkeit der 3D Oberfläche der durch Catch ${ }^{\circledR}$ generierten 3D Modelle gegenüber dem Vectra ${ }^{\circledR}$ Referenzmodel untersucht. Dabei wurden auch die 3D Aufnahmen zwischen Smartphone und Tablet verglichen.

Zum 3D Oberflächenvergleich berechnet die Software MIRROR ${ }^{\circledR}$ anhand automatischer Überlagerung des Vectra ${ }^{\circledR}$ Referenzmodels mit den zu vergleichenden 3D Modellen von Catch ${ }^{\circledR}$ (engl. superimposition $[16,17])$ die Gesamtabweichung beider Oberflächen. Das Ergebnis dieser Oberflächen (surface-to-surface)Analyse wird als quadratisches Mittel (engl. root mean square, RMS $[18,19]$ ) in Millimetern angegeben.

Für eine differenziertere Analyse der verschiedenen Einstellungen von Catch ${ }^{\circledR}$ und deren Auswirkung auf das 3D Model, wurde das Gesicht in modifizierte Ästhetische Einheiten (Stirn - zentral und lateral, Nase, Wange - medial und lateral, Mund und Kinn Region) nach Fattahi et al. [20] gegliedert ( $\bullet$ Abb. 3).

Insgesamt wurden 30 Catch $^{\circledR}$ Modelle, generiert durch Smartphone und Tablet Aufnahmen aus je 5 Bildersätzen (16, 12, 9, 6 und 3 Bilder) und den 3 Qualitätsstufen $\mathrm{HQ}$, SQ LQ mit dem Vectra ${ }^{\circledR}$ Referenzmodel verglichen. Die Benennung der einzel-
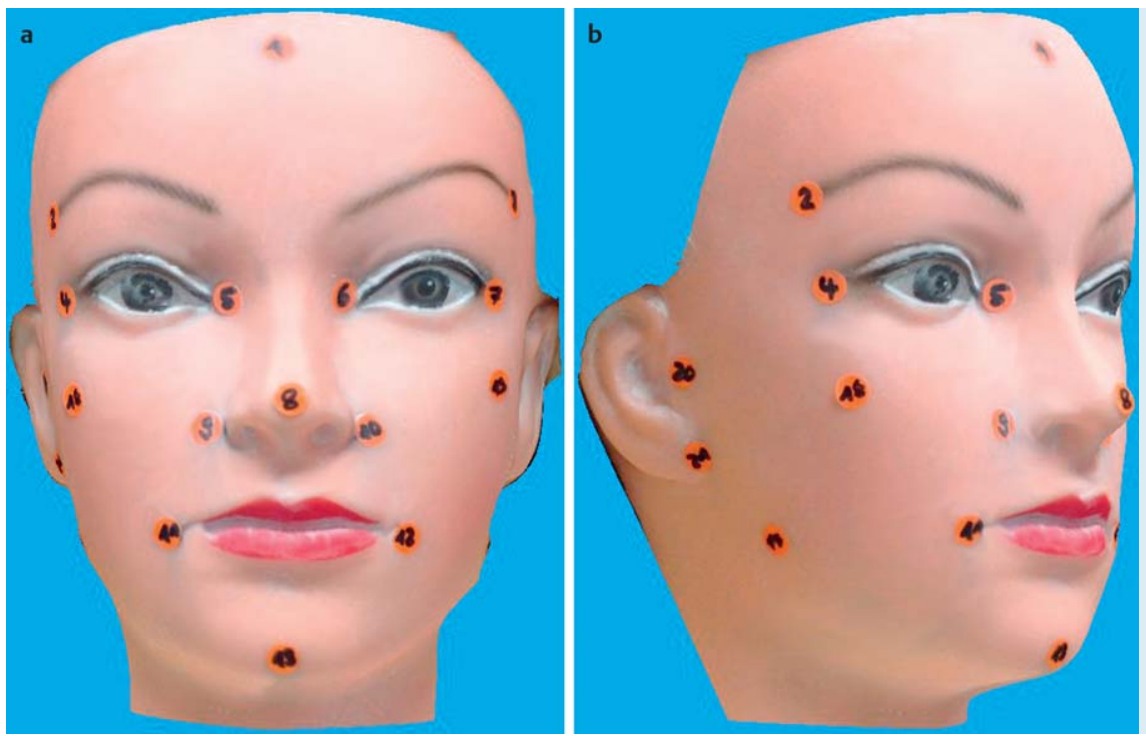

Abb. 2 a + b: Landmarken. Insgesamt 21 Landmarken wurden im Gesichtsbereich gesetzt. 
Tab. 1 Distanzen. Übersicht der durch Landmarken definierten Distanzen.

\begin{tabular}{|c|c|c|}
\hline \multirow[t]{2}{*}{ Beschreibung der Distanzen } & \multicolumn{2}{|l|}{ Landmarken } \\
\hline & Rechte Seite & Linke Seite \\
\hline Stirn - Augenbraue lateral & 1 und 2 & 1 und 3 \\
\hline $\begin{array}{l}\text { Augenbraue lateral - lateraler } \\
\text { Augenwinkel }\end{array}$ & 2 und 4 & 3 und 7 \\
\hline medialer Augenwinkel - Nasenflügel & 5 und 9 & 6 und 10 \\
\hline Nasenflügel - Mundwinkel & 9 und 11 & 10 und 12 \\
\hline Nasenflügel - Jochbogen & 9 und 18 & 10 und 19 \\
\hline Nasenspitze - Nasenflügel & 8 und 9 & 8 und 10 \\
\hline Mundwinkel - Kinn & 11 und 13 & 12 und 13 \\
\hline Augenbraue lateral - Tragus & 2 und 20 & 3 und 16 \\
\hline Augenwinkel lateral - Tragus & 4 und 20 & 7 und 16 \\
\hline Augenwinkel lateral - Jochbogen & 4 und 18 & 7 und 19 \\
\hline Augenwinkel medial - Jochbogen & 5 und 18 & 6 und 19 \\
\hline Jochbogen - Mundwinkel & 11 und 18 & 12 und 19 \\
\hline Jochbogen - Tragus & 18 und 20 & 16 und 19 \\
\hline Jochbogen - Ohrläppchen & 18 und 21 & 17 und 19 \\
\hline Jochbogen - Kieferwinkel & 14 und 18 & 15 und 19 \\
\hline Kieferwinkel - Kinn & 13 und 14 & 13 und 15 \\
\hline Kieferwinkel - Mundwinkel & 11 und 14 & 12 und 15 \\
\hline Tragus - Ohrläppchen & 20 und 21 & 16 und 17 \\
\hline Kieferwinkel - Ohrläppchen & 14 und 21 & 15 und 17 \\
\hline Mundwinkel - Tragus & 11 und 20 & 12 und 16 \\
\hline Stirn - Nasenspitze & \multicolumn{2}{|c|}{1 und 8} \\
\hline Augenbraue lateral rechts - links & \multicolumn{2}{|c|}{2 und 3} \\
\hline medialer Augenwinkel rechts - links & \multicolumn{2}{|c|}{5 und 6} \\
\hline Nasenspitze - Kinn & \multicolumn{2}{|c|}{8 und 13} \\
\hline
\end{tabular}

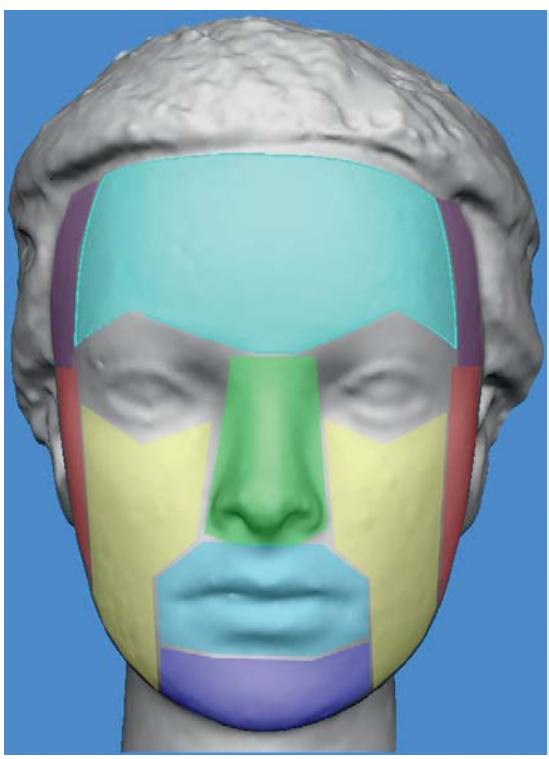

Abb. 3 Einteilung in ästhetische Einheiten. Das Gesicht wurde unterteilt in zentrale (türkis) und laterale (lila) Stirn, nasal (grün), mediale (gelb) und laterale (rot) Wange, sowie in Mund (hellblau) und Kinn (dunkelblau). Die gewählten Areale wurden anhand weiterer Landmarken automatisch durch die MIRROR ${ }^{\circledR}$ Software auf alle Modelle gleich übertragen.

nen Modelle erfolgte nach ihrer Bilderanzahl und der Qualitätsstufe.

\section{Aufnahme und Verarbeitungszeit}

Für jeweils eine 3D Aufnahme mittels Vectra ${ }^{\circledR}$ und Catch ${ }^{\circledR}$ System wurde die Zeit vom Beginn der Aufnahme bis zum finalen 3D Model festgehalten. Die Gesamtzeit wurde weiter unterteilt in eine Akquisitions- und Verarbeitungsphase. Die Akquisitionsphase beinhaltet die Zeit zur Aufnahme der 16 Bilder. Darauf folgt die Verarbeitungsphase bis zum fertigen 3D Model.

\section{Statistik}

Zur statistischen Auswertung wurde das Programm IBM ${ }^{\circledR}$ SPSS $^{\circledR}$ Statistics 19 (SPSS Inc., IL, USA) genutzt.

Die Auswertung der Distanzmessungen wurden als absolute Abweichungen in Millimeter [mm] und relative Abweichungen in Prozent [\%] wiedergegeben.

Alle Distanzmessungen wurden mit dem Wilcoxon-MannWhitney-Test verglichen und der Pearson Korrelationskoeffizient wurde bestimmt. Die Wahrscheinlichkeit, dass sich die Messungen signifikant unterscheiden, wurde mit $\mathrm{p}<0,05$ angenommen.

\section{Ergebnisse}

\section{Genauigkeitsanalyse}

Der Vergleich zwischen manueller Maßbandmessung und der digitalen Auswertung der 3 Catch Modelle aus 16 Bildern zeigte keinen signifikanten Unterschied, mit den geringsten Abweichungen für die Standardqualität (HQ $p=0,765 ; 0,69 \pm 0,20 \mathrm{~mm}$; SQ $p=0,917 ; 0,512 \pm 0,12 \mathrm{~mm}$; LQ $\mathrm{p}=0,913 ; 0,66 \pm 0,14 \mathrm{~mm}$ ). - Abb. 4 vergleicht die absolute Abweichung der Catch ${ }^{\circledR}$ Standard Qualität (SQ) und dem Vectra ${ }^{\circledR}$ Model gegenüber der manuellen Maßbandmessung. Die digitalen Distanzen der 3 Modelle zeigten eine hohe Korrelation zu den manuellen Maßbandmessungen $(r=0,999)$. Die größten Abweichungen zeigten sich in den Distanzen zwischen Mundwinkel außen und Tragus (HQ: 2,31 $\pm 0,21 \mathrm{~mm}$; SQ: 1,53 $\pm 0,14 \mathrm{~mm}$; LQ: $2,51 \pm 0,20 \mathrm{~mm}$ ), sowie zwischen Kieferwinkel und Kinn (HQ: 2,95 $\pm 0,21 \mathrm{~mm}$; SQ:

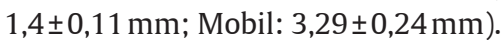

Die digitale Messung der Vectra Modelle zeigte keine signifikanten Unterschiede gegenüber der manuellen Messung $(p=0,967$; $0,353 \pm 0,12 \mathrm{~mm})$.

Es zeigten sich geringe Unterschiede in der 3D Aufnahmen mittels Catch ${ }^{\circledR}$ zwischen dem Smartphone und Tablet mit einer durchschnittlichen Abweichung von 0,126 mm $\pm 0,02 \mathrm{~mm}$ RMS ( $\min 0,079 \mathrm{~mm}$, max 0,178 mm).

Die Auswirkung auf die Genauigkeit der Oberflächenrekonstruktion durch unterschiedliche Einstellungen wurde in $\bullet$ Abb. 5 zusammengefasst.

Hierbei zeigten sich keine signifikanten Unterschiede der RMS Abweichungen zwischen den Modellen aus 16, 12 und 9 Bildern mit ihren 3 Qualitätsstufen ( $P>0,05)$. Es ergaben sich insgesamt niedrigere Gesamtabweichungen für Modelle mit hoher Verarbeitungsqualität (HQ) gegenüber den Standard (SQ) und niedrigen (LQ). Modelle aus 6 und 3 Bildern unterschieden sich jedoch signifikant gegenüber den vorherig genannten $(P<0,001)$. Die Ergebnisse der Messung mit 6 und 3 Bildern repräsentieren jene Teile, deren Auswertung möglich war.

Der Vergleich zwischen ästhetischen Einheiten zeigte niedrigere RMS Abweichungen für zentrale und mediale Regionen (Stirn zentral, Nase, Wange medial, Kinn; durchschnittlich 0,43 $\pm 0,16 \mathrm{~mm}$ RMS; $\bullet$ Abb. 6a) im Vergleich zu lateralen Regionen (Stirn lateral, Wange lateral; 0,73 $\pm 0,22 \mathrm{~mm}$ RMS; $\bullet$ Abb. 6b).

\section{Aufnahme und Verarbeitungszeit}

Das Vectra System erstellte ein 3D Model in insgesamt 2 min, mit einer Akquisitionszeit von $3 \mathrm{~ms}$. Im Vergleich benötigte Catch die 5-fache Zeit (10 min) bis zum finalen Model, mit einer Akquisitionszeit von 1 min zur Aufnahme der 16 Bilder mit der mobilen 


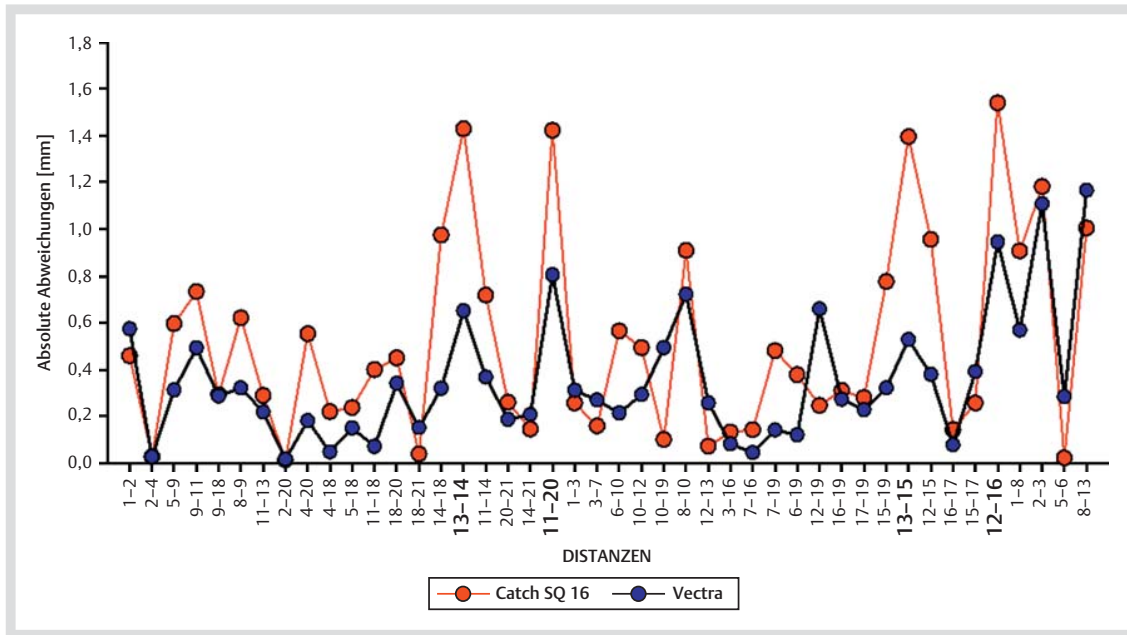

Abb. 4 Vergleich der Distanzmessungen. Dargestellt sind die absoluten Abweichungen der digitalen Messungen am Catch ${ }^{\circledR}$ 16SQ (aus 16 Bildern, Standard Qualität) Model und Vectra ${ }^{\circledR}$ Referenzmodel gegenüber der manuellen Maßbandmessung. Es zeigten sich insgesamt niedrigere Abweichungen des Vectra Systems gegenüber dem Catch Model, bei tendenziell gleichem Abweichungsverhalten. Beide digitale Messungen unterschieden sich jedoch nicht signifikant gegenüber der manuellen Messung.

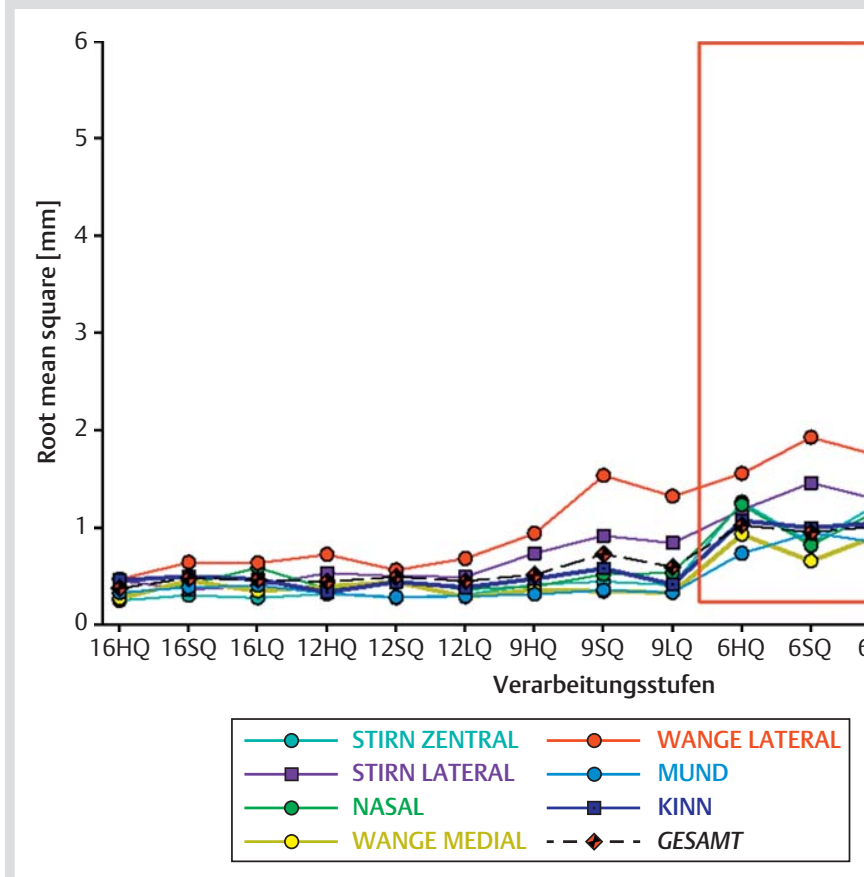

Abb. 5 Auswirkung unterschiedlicher Einstellungen. Dargestellt sind verschiedene Einstellungen aus Bilderanzahl und Verarbeitungsqualität (hoch $\mathrm{HQ}$, Standard SQ, niedrig LQ) mit ihrer Oberflächenabweichung (mm RMS) gegenüber dem Vectra ${ }^{\circledR}$ Referenzmodel insgesamt, sowie für die einzelnen ästhetischen Einheiten. Aufnahmen mit 6 und 3 Bildern sind für das Verfahren nicht zu empfehlen (rot eingerahmt). Laterale Abschnitte zeigten insgesamt höhere Abweichungen.

Anwendung ( $\bullet$ Abb. 7). Eine Reduktion der Bilderzahl verkürzte die Akquisitionszeit für 12 (40 s), 9 (30 s), 6 (20 s) und 3 Bilder (10 s), die Verarbeitungszeit blieb konstant bei durchschnittlich $9 \mathrm{~min}$.

\section{Diskussion}

$\nabla$

Unsere Ergebnisse mit der Applikation Autodesk 123d Catch ${ }^{\circledR}$ zeigen einen neuartigen, kostengünstigen Ansatz zur 3D Rekonstruktion aus Bildern mit einem Smartphone und Tablet.

Im ersten Teil der Genauigkeitsanalyse zeigten die digitalen Distanzmessungen der 3 Catch ${ }^{\circledR}$ Modelle geringe, nicht signifikante Unterschiede gegenüber der manuellen Messung. Insbesondere die Distanzen der Standard Qualität ergaben vergleichbar genaue Ergebnisse mit dem Vectra ${ }^{\circledR}$ System. Größere Abweichungen zeigten sich bei längeren Distanzen zwischen der manuellen und digitalen Messung, nicht jedoch zwischen beiden digitalen Systemen. Eine mögliche Erklärung bietet die individuelle Ungenauigkeit bei der manuellen Maßbandmessung zwischen weit entfernten Punkten. Auch zeigten sich nicht die in früheren $\mathrm{Pu}-$ blikationen typischen Messungenauigkeiten [3,6,13,14,21] zwischen Nase und Kinn, da die verwendete Puppe mit vereinfachter Mundregion eine genauere manuelle Messung zulässt. Eine zukünftige Messung an Probanden sollte voraussichtlich größere Abweichungen aufweisen.

Im zweiten Teil zeigten sich mittels 3D Oberflächenüberlagerung des Vectra ${ }^{\circledR}$ Referenzmodels mit den Catch ${ }^{\circledR}$ Modellen generell bessere Ergebnisse für zentrale und mediale ästhetische Einheiten in allen Verarbeitungsstufen mit 16, 12 und 9 Bildern. Die digitale Photogrammetrie beruht auf der Übereinstimmung gleicher Punkte auf verschiedenen Bildern und berechnet daraus ein 3D Model. Je mehr Bilder auf einen Bereich gerichtet sind, umso mehr profitiert der Algorithmus für die 3D Rekonstruktion. In unserem Versuchsaufbau waren die ästhetischen Einheiten von Nase, zentraler Stirn und Kinn auf allen Bildern vorhanden, was die geringere Abweichung erklären kann. Laterale Anteile zeigten höhere Abweichungen vom Referenzmodel, da sie insgesamt auf weniger Aufnahmen vorhanden waren. 


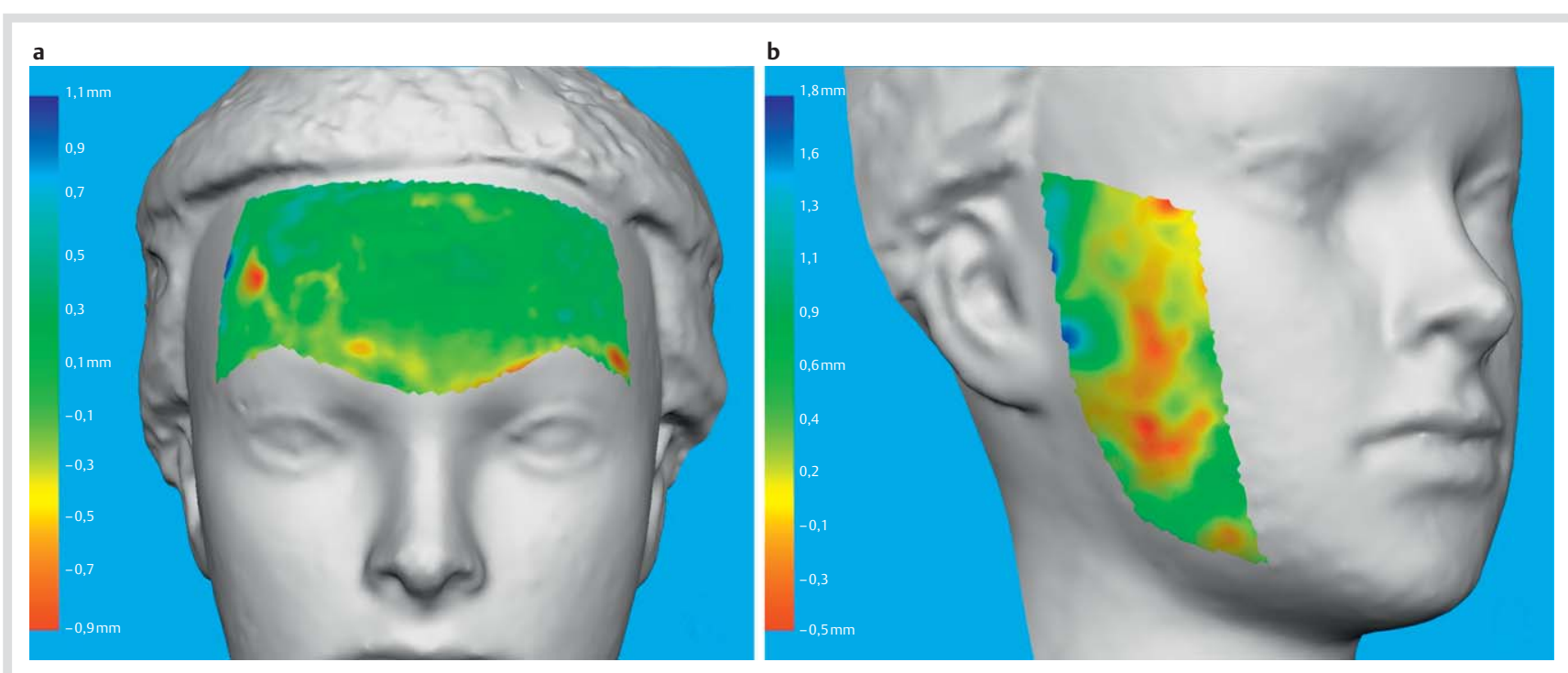

Abb. 6 a +b Oberflächenabweichung vom Referenzmodel. a - Oberflächenabweichung der zentralen Stirneinheit des Catch SQ Models mit 16 Bildern (16SQ) mit einer niedrigen Abweichung von 0,25 mm RMS. b - Laterale Wangeneinheit des aus 12 Bildern in niedriger (LQ) Qualität (12LQ) generierten Catch Models mit einer erhöhten Abweichung von 0,68 mm RMS.

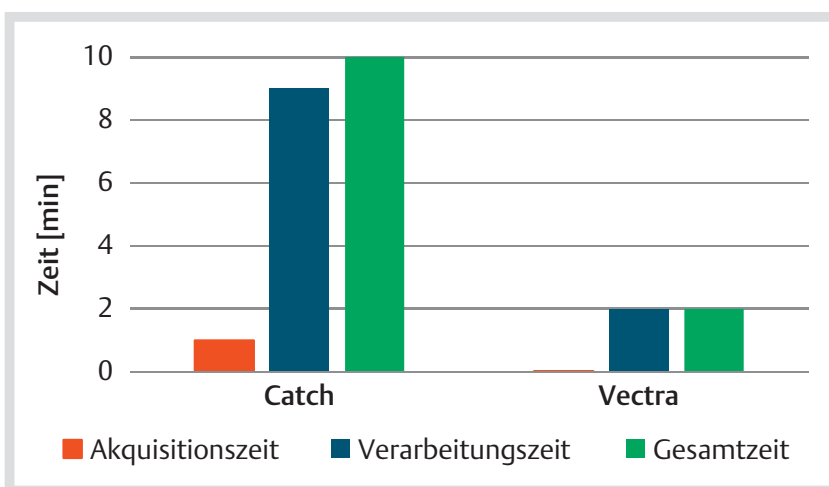

Abb. 7 Zeiten. Akquisitions-, Verarbeitungs- und Gesamtzeit der 3D Oberflächenerfassung und Verarbeitung für das Vectra Referenzmodel und einem Catch Model aus 16 Bildern in hoher Qualität (HQ). Bedeutend ist eine kurze Akquisitionszeit.

Wir untersuchten durch die nachträgliche selektive Reduzierung der Bilderanzahl und Einstellung der 3 Verarbeitungsqualitäten HQ, SQ und LQ die Auswirkungen auf das generierte 3D Model. Dadurch konnte mit identischen Aufnahmen gearbeitet und Ungenauigkeiten bei erneuten Aufnahmesitzungen vermieden werden. Der Studienaufbau mit Puppenkopfmodel zeigte akzeptable Ergebnisse [19,22,23] für die Generierung von 3D Modellen mit 16, 12 und 9 Bildern (RMS $\min 0,378 \mathrm{~mm}$, max. $0,781 \mathrm{~mm}$ ). Es zeigten sich nur geringe Unterschiede in der Konfiguration aus 16, 12 und 9 Bildern für mediale ästhetische Einheiten und tendenziell steigende Abweichungen insbesondere für die lateralen Einheiten. Verarbeitungen in hoher Qualität (HQ) hatten gegenüber der SQ und LQ Qualität niedrigere totale RMS Werte. Die Ergebnisse verdeutlichen, dass für eine ausreichende, gleichmäßige 3D Rekonstruktion mindestens 9 Bilder benötigt werden. Die Auswertung von 6 und 3 Bildern zeigte starke Abweichungen vom Referenzmodel und zahlreiche Oberflächenlöcher durch fehlerhafte Verarbeitung. Somit konnten nur vorhandene Bereiche ausgewertet werden, wodurch die erhaltenen Ergebnisse zu unterschätzen sind.
Die 3D Rekonstruktion beschrieben durch Oliveira-Santos et al. [9] erreichte für das Gesicht Abweichungen von durchschnittlich $1 \mathrm{~mm}$ mit maximalen Abweichungen unter $2 \mathrm{~mm}$. Dabei wurden mit nur 3 Bildern einer Digitalkamera die Gesichter von Probanden rekonstruiert. Zusätzlich wurde auch der komplizierte Augenbereich zur Auswertung miteinbezogen. Somit lassen sich die Ergebnisse nur bedingt vergleichen, da von einer vereinfachten Aufnahme am Puppenmodel auszugehen ist und Catch keine ausreichenden 3D Modelle mit nur 3 Bildern generieren konnte. Obwohl das Smartphone und Tablet der Firma Apple ${ }^{\circledR}$ baugleiche Kameraeinheiten für ihre Aufnahmen nutzen, wäre eine größere Abweichung durch die Software oder den Untersucher trotzt standardisiertem Aufbau zu erwarten. Catch ${ }^{\circledR}$ erfasste mit beiden Geräten 3D Modelle mit einer geringen Abweichung zueinander (durchschnittlich 0,126 $\pm 0,04 \mathrm{~mm}$ RMS), weshalb wir beide Geräte für den Einsatz zur 3D Fotografie als gleichwertig ansehen. Ein Unterschied zeigte sich nur in der Form und Haptik der Geräte. Ein größeres Display erleichtert initial die Aufnahme bei ungeübten Untersuchern, jedoch bietet das Smartphone bei gleicher Qualität eine einhändige Benutzung und wird im klinischen Alltag häufiger gegenüber Tablets eingesetzt. Beide Geräte zeichnen sich mit der intuitiven Anwendung von Catch ${ }^{\circledR}$ durch ihre schnelle und anwenderfreundliche Bedienung aus.

$\mathrm{Zu}$ Bedenken ist, dass die Qualität der Aufnahmen durch beide Geräte bereits zum Zeitpunkt der Studie im Vergleich zu professionellen Digitalkameras und auch hochpreisigen mobilen Endgeräten nur durchschnittliche Auflösungen ( $5 \mathrm{mp}$ ) erzielten. Da die Pixeldichte, also das Auflösungsvermögen pro Bild, mit der späteren Verarbeitung korreliert, erreichen Professionelle Kamerasysteme mit qualitativ hochwertigen Kameras (36mp) genauere 3D Modelle. Mit zunehmender Verbesserung der Kameras von Smartphones und Tablets wird auch die Qualität der 3D Erfassung erhöht.

In Verbindung mit den geringen Unterschieden zwischen den Aufnahmen mit 16,12 und 9 Bildern sollten mit der bisherigen Technik weniger Bilder favorisiert werden, um die initiale Akquisitionsphase zu verkürzen. Diese Phase, die benötigt wird um alle Fotos des Objektes aufzunehmen, ist als einzige anfällig für 
Störgrößen und wirkt sich unabhängig von verschiedenen Einstellungen negativ bei falscher Durchführung auf die Objektqualität aus. Die spätere Verarbeitungsphase auf externen Servern oder des Vectra ${ }^{\circledR}$ Systems wird vornehmlich durch die Rechenleistung derer bestimmt und könnte sich durch bessere Hardware beschleunigen lassen. Im Hinblick auf weitere Studien mit Probanden ist eine kurze Aufnahmezeit anzustreben, um die Auswirkung durch unwillkürliche zu reduzieren. Das Vectra ${ }^{\circledR}$ System und andere professionelle Kamerasysteme minimieren durch ihre einzeitigen Aufnahmen im Bereich von Millisekunden diese Problematik. Jedoch limitieren die meist große Bauweise und räumlich eingegrenzte Oberflächenerfassung den Einsatz auf ausgewählte Körperregionen und erlauben eine $360^{\circ}$ Erfassung von Körpern nur durch mehrmalige Aufnahmen und nicht automatisierte Zusammenfügung und sind somit auch anfälliger für Störfaktoren. Mit einer erhöhten Bilderanzahl von bis zu 70 Aufnahmen lassen sich jedoch mit der mobilen Anwendung Catch ${ }^{\circledast}$ ganze Körper erfassen, deren Qualität in weiteren Studien zu untersuchen ist.

Obwohl eine externe 3D Verarbeitung den technischen Aufwand für den behandelnden Arzt reduziert, stellt die Weitergabe sensibler Patientendaten ein Datenschutzrisiko dar. Für einen zukünftigen Einsatz einer solchen Technik sind die Klärung rechtlicher Fragen, sowie ein zuverlässiger Schutz gegenüber dem Datenverlust zu gewährleisten.

\section{Zusammenfassung}

$\nabla$

Insgesamt konnten durch den standardisierten Ansatz mit der mobilen Applikation 123 Catch $^{\circledR}$ am Puppenmodel eine zufriedenstellende Genauigkeit der 3D Rekonstruktion gegenüber der manuellen Distanzmessung und dem Oberflächenvergleich mit einem Vectra ${ }^{\circledR}$ Referenzmodel gezeigt werden. In Verbindung mit der Nutzung mobiler Geräte wie Smartphones und Tablets zeigte sich eine schnelle, intuitive Lösung zur 3D Oberflächenerfassung. Eine Fehlerquelle stellt hierbei die verlängerte Akquisitionszeit durch eine erhöhte Anzahl an Fotos dar. Bei geringen Anschaffungskosten der Geräte kann die kostenlos erhältliche Anwendung in Zukunft eine bedeutende Rolle darstellen.

Weitere Studien an Probanden werden benötigt, um den Einfluss von unwillkürlichen Bewegungen wie der Atmung, als auch einer komplexeren Oberflächenstruktur zu untersuchen.

\section{Danksagung}

Besonderer Dank gilt der der Forschungsgruppe CAPS - Computer Aided Plastic Surgery, Klinik für Plastische Chirurgie und Handchirurgie, Klinikum rechts der Isar, Technische Universität München, insbesondere Prof. Dr. L. Kovacs, Dr. M. Eder und Dipl.Ing. A. Volf für Ihre fachlichen Anregungen, welche durch die ausgewiesene Kenntnisse auf dem Gebiet der 3-D Technologie einen enormen Beitrag zur Realisierung dieser Arbeit darstellten.

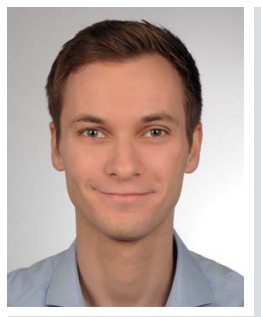

Konstantin Christoph Koban geb. 1989 in Berlin. Seit 2008 Studium der Humanmedizin an der Ludwig-Maximilians-Universität München. Doktorand der Arbeitsgruppe „Dreidimensionale (3-D) Fotovolumetrie“ (Dr. Leitsch, PD Dr. Holzbach) der Handchirurgie, Plastische Chirurgie und Ästhetische Chirurgie an der Ludwig-Maximilians-Universität München (Prof. Giunta).

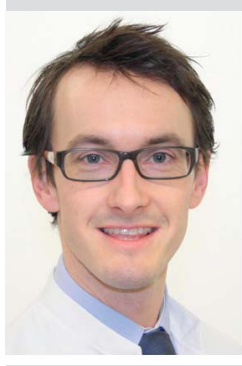

Dr. med. Sebastian Leitsch geb. 1979 in Herne. Studium der Humanmedizin an der Universität Witten/ Herdecke mit Studienaufenthalten in Houston, Kapstadt und Zürich. Dissertation zur genomischen Analyse von Nierentumorentitäten mittels der komparativen genomischen Hybridisierung und Mikrosatellitenanalyse unter Prof. Störkel an der Universität Witten/ Herdecke. Von 2007 bis 2008 Assistenzarzt an der Klinik für Chirurgie am akademischen Lehrkrankenhaus Männedorf der Universität Zürich (Prof. Hollinger). Bis 2011 an der Klinik für Plastische Chirurgie am Klinikum Köln-Merheim der Universität Witten/Herdecke (Prof. Spilker). Seit 2012 an der Handchirurgie, Plastische Chirurgie und Ästhetische Chirurgie an der Ludwig-Maximilians-Universität München (Prof. Giunta).

Interessenkonflikt: Nein

\section{Literatur}

1 Deacon AT, Anthony AG, Bhatia SN et al. Evaluation of a CCD-based facial measurement system. Med Inform (Lond) 1991; 16: 213-228

2 Galdino GM, Nahabedian M, Chiaramonte $M$ et al. Clinical applications of three-dimensional photography in breast surgery. Plast Reconstr Surg 2002; 110: 58-70

3 Tepper OM, Small K, Rudolph L et al. Virtual 3-dimensional modeling as a valuable adjunct to aesthetic and reconstructive breast surgery. Am J Surg 2006; 192: 548-551

4 Tepper OM, Karp NS, Small $K$ et al. Three-dimensional imaging provides valuable clinical data to aid in unilateral tissue expander-implant breast reconstruction. Breast J 2008; 14: 543-550

5 Tzou CH, Frey M. Evolution of 3D surface imaging systems in facial plastic surgery. Facial Plast Surg Clin North Am 2011; 19: 591-602 vii

6 Spanholtz TA, Leitsch S, Holzbach T et al. 3-dimensional imaging systems: first experience in planning and documentation of plastic surgery procedures. Handchir Mikrochir Plast Chir 2012; 44: 234-239

7 Wheat JS, Choppin S, Goyal A. Development and assessment of a Microsoft Kinect based system for imaging the breast in three dimensions. Med Eng Phys 2014 DOI:

8 Henseler H, Kuznetsova A, Vogt P et al. Validation of the Kinect device as a new portable imaging system for three-dimensional breast assessment. J Plast Reconstr Aesthet Surg 2014 DOI:

9 Oliveira-Santos T, Baumberger C, Constantinescu M et al. 3D face reconstruction from 2D pictures: first results of a web-based computer aided system for aesthetic procedures. Ann Biomed Eng 2013; 41: 952-966

10 de Heras Ciechomski P, Constantinescu M, Garcia J et al. Development and implementation of a web-enabled 3D consultation tool for breast augmentation surgery based on 3D-image reconstruction of 2D pictures. Journal of medical Internet research 2012; 14: e21

11 Workman $A D$, Gupta SC. A plastic surgeon's guide to applying smartphone technology in patient care. Aesthet Surg J 2013; 33: 275-280

12 Mohan AT, Branford OA. iGuide to plastic surgery: iPhone apps, the plastic surgeon, and the health care environment. Aesthet Surg J 2012; 32: 653-658 
13 Aldridge K, Boyadjiev SA, Capone GT et al. Precision and error of threedimensional phenotypic measures acquired from $3 \mathrm{dMD}$ photogrammetric images. Am J Med Genet A 2005; 138A: 247-253

14 Kovacs L, Zimmermann A, Brockmann G et al. Accuracy and precision of the three-dimensional assessment of the facial surface using a 3-D laser scanner. IEEE Trans Med Imaging 2006; 25: 742-754

15 Wong JY, Oh AK, Ohta E et al. Validity and reliability of craniofacial anthropometric measurement of 3D digital photogrammetric images. Cleft Palate Craniofac J 2008; 45: 232-239

16 Hoefert CS, Bacher $M$, Herberts T et al. Implementing a superimposition and measurement model for 3D sagittal analysis of therapy-induced changes in facial soft tissue: a pilot study. J Orofac Orthop 2010; 71: 221-234

17 Maal TJ, van Loon B, Plooij JM et al. Registration of 3-dimensional facial photographs for clinical use. J Oral Maxillofac Surg 2010; 68: 2391-2401

18 Fourie Z, Damstra J, Gerrits PO et al. Evaluation of anthropometric accuracy and reliability using different three-dimensional scanning systems. Forensic Sci Int 2011; 207: 127-134
19 Eder M, Brockmann G, Zimmermann A et al. Evaluation of precision and accuracy assessment of different 3-D surface imaging systems for biomedical purposes. J Digit Imaging 2013; 26: 163-172

20 Fattahi TT. An overview of facial aesthetic units. J Oral Maxillofac Surg 2003; 61: 1207-1211

21 Papadopoulos MA, Jannowitz C, Boettcher $P$ et al. Three-dimensional fetal cephalometry: an evaluation of the reliability of cephalometric measurements based on three-dimensional CT reconstructions and on dry skulls of sheep fetuses. J Craniomaxillofac Surg 2005; 33: 229-237

22 Naudi KB, Benramadan R, Brocklebank L et al. The virtual human face: superimposing the simultaneously captured 3D photorealistic skin surface of the face on the untextured skin image of the CBCT scan. Int J Oral Maxillofac Surg 2013; 42: 393-400

23 Patete $P$, Eder M, Raith $S$ et al. Comparative assessment of 3D surface scanning systems in breast plastic and reconstructive surgery. Surg Innov 2013; 20: 509-515 